\title{
Messagers intracellulaires produits par la phospholipase $C$
}

Les agents mobilisateurs du calcium agissent en stimulant la phospholipase C qui, en hydrolysant un phospholipide membranaire, produit le diacylglycérol et l'inositol 1,4,5-trisphosphate, deux messagers intracellulaires dont le rôle et le mode d'action sont relativement bien connus. L'inositol 1,4,5-trisphosphate se lie à un récepteur spécifique qui contrôle l'activité d'un canal calcique situé sur la membrane d'un réservoir intracellulaire de calcium. Le diacylglycérol stimule la protéine kinase $\mathbf{C}$. A l'intérieur d'une cellule stimulée, l'inositol 1,4,5-trisphosphate subit une série d'étapes de phosphorylation et de déphosphorylation qui produisent différents composés dont le rôle physiologique n'est, à ce jour, pas encore bien défini.

\section{Gaétan Guillemette}

\section{ADRESSE}

G. Guillemette : professeur adjoint. Département de pharmacologie, faculté de médecine, université de Sherbrooke, Sherbrooke, Qué- es cellules communiquent entre elles par le biais d'agents externes (hormones, neurotransmetteurs, autacoïdes) qui parcourent des distances plus ou moins grandes dans les liquides biologiques pour porter leurs messages. Ces signaux externes sont détectés par des récepteurs à la surface des cellules cibles et traduits en un répertoire restreint de seconds messagers intracellulaires. Parmi les plus connus de ces seconds messagers, l'inositol 1,4,5-trisphosphate (Ins 1,4,5- $\mathrm{P}_{3}$ ) et le diacylglycérol (DG) sont les composants actifs d'un mécanisme de transduction qui a attiré beaucoup d'attention au cours des dernières années [1-3]. A partir de l'observation des Hokins, dans les années 1950, que la stimulation de la sécrétion pancréatique par certains agonistes augmente le métabolisme des phospholipides de l'inositol [4], en passant par l'hypothèse de Michell, en 1975, selon laquelle ce phénomène serait relié à la mobilisation du
$\mathrm{Ca}^{2+}[5]$, les résultats issus de nombreux laboratoires, incluant ceux de Nishizuka [6] et de Berridge [7, 8], ont permis de définir les principales étapes de ce mécanisme d'action. Une projection simplifiée de ce mécanisme (modèle présenté sur la figure 1) montre que le récepteur, par l'intermédiaire d'une G-protéine [9], contrôle l'activité d'une phosphodiestérase membranaire (la phospholipase C) qui coupe un phospholipide du feuillet interne de la membrane plasmique (le phosphatidylinositol 4,5-bisphosphate, $\mathrm{PIP}_{2}$ ) pour produire, d'une part, le DG et, d'autre part, l'Ins 1,4,5- $\mathrm{P}_{3}$. Le DG est un lipide neutre qui demeure dans le plan de la membrane plasmique et qui active la protéine kinase $C[10$, 11]. L'Ins $1,4,5-\mathrm{P}_{3}$ est un produit polaire qui diffuse dans le cytosol et contrôle l'activité d'un canal calcique situé sur un réservoir intracellulaire de $\mathrm{Ca}^{2+}$. Le $\mathrm{Ca}^{2+}$ ainsi libéré active des protéines spécifiques (troponine, parvalbumine, calmoduline, etc.) qui, elles-mêmes, activent des 
processus spécifiques (entre autres la protéine kinase dépendante du calcium et de la calmoduline). Les effets combinés des voies activées par le DG et l'Ins $1,4,5-\mathrm{P}_{3}$ conduisent à la réponse biologique finale. L'analyse détaillée de chacune des étapes de ce mécanisme d'action a fait l'objet d'efforts considérables au cours des dernières années. L'un des sujets qui a attiré beaucoup d'attention est le mécanisme par lequel l'Ins 1,4,5- $\mathrm{P}_{3}$ augmente le $\mathrm{Ca}^{2+}$ intracellulaire.

\section{Rôle de l'Ins 1,4,5-P}

La relation entre l'hydrolyse des phospholipides de l'inositol et l'homéostasie calcique n'a été confirmée que lors de la découverte du rôle de l'Ins 1,4,5- $\mathrm{P}_{3}$. La première observation faite avec des cellules acineuses pancréatiques perméabilisées (dans un milieu pauvre en $\mathrm{Ca}^{2+}$ ) a démontré que le $\mathrm{Ca}^{2+}$ accumulé dans le réticulum endoplasmique, par un mécanisme dépendant de l'ATP, est rapidement relâché suite à l'addition de faibles quantités d'Ins 1,4,5- $\mathrm{P}_{3}$ [7]. L'effet semble spécifique puisque l'Ins $1,4-\mathrm{P}_{2}$ et l'Ins 1-P sont inactifs. Les concentrations nécessaires pour mobiliser le $\mathrm{Ca}^{2+}(0,2-1,0 \mu \mathrm{M})$ sont compatibles avec les niveaux atteints lors d'une stimulation cellulaire. Le $\mathrm{Ca}^{2+}$ libéré par l'Ins $1,4,5-\mathrm{P}_{3}$ ne provient pas des mitochondries, puisque l'expérience fut faite en absence de substrats métaboliques et en présence d'inhibiteurs mitochondriaux (à ce sujet, il est maintenant reconnu que la mitochondrie n'est pas impliquée dans la régulation des niveaux de $\mathrm{Ca}^{2+}$ à l'intérieur d'une cellule saine dans des conditions physiologiques normales [12]). Le $\mathrm{Ca}^{2+}$ est séquestré dans une structure vésiculaire par un mécanisme dépendant de l'ATP et du $\mathrm{Mg}^{2+}$ suggérant que le site d'action de l'Ins $1,4,5-\mathrm{P}_{3}$ est le réticulum endoplasmique [7]. Les résultats de cette expérience originale furent reproduits dans de nombreux laboratoires au moyen de plusieurs types de cellules (pour revue, voir $[1,3]$ ).

Des études de structure-activité ont démontré que les groupements phosphates situés sur les carbones voisins en position 4 et 5 sont essentiels pour la libération $\mathrm{du} \mathrm{Ca}^{2+}$. Le groupement phosphate en position l n'est pas essentiel, puisque l'Ins $4,5-\mathrm{P}_{2}$ ou $\mathrm{m} / \mathrm{s} n^{\circ} 8 \mathrm{vol} .5$, octobre 89
l'Ins 2,4,5- $\mathrm{P}_{3}$ peuvent provoquer la libération de $\mathrm{Ca}^{2+}$ mais à des doses beaucoup plus fortes. Ces résultats sont compatibles avec la notion d'un récepteur pour l'Ins 1,4,5- $\mathrm{P}_{3}$. L'hypothèse de l'existence d'un récepteur fut appuyé par la mise en évidence de sites de liaison pour l'Ins 1,4,5- $\mathrm{P}_{3}$ dans le cortex surrénalien [13], le foie [14] et autres tissus périphériques[15-17]. La liaison du ${ }^{32} \mathrm{P}$ Ins 1,4,5- $\mathrm{P}_{3}$ est spécifique, saturable et proportionnelle à la quantité de microsomes ajoutée. Les cinétiques rapides d'association et de dissociation sont compatibles avec les mouvements rapides de $\mathrm{Ca}^{2+}$ induits par l'Ins $1,4,5-\mathrm{P}_{3}$ dans les cellules perméabilisées. Les études de structureactivité ont montré que les sites de liaison possèdent les mêmes critères de sélectivité décrits dans les expériences de mobilisation de $\mathrm{Ca}^{2+}$. Il faut noter que ces études sont limitées par le nombre restreint d'analogues structuraux de l'Ins $1,4,5-\mathrm{P}_{3}$ préparés par les méthodes biochimiques. Cependant des rapports récents décrivant la synthèse chimique de ces produits [18, 19] laissent espérer pour un avenir rapproché la disponibilité de nouveaux analogues en quantités appréciables.

Un critère important, afin de s'assurer que les sites de liaison pour l'Ins $1,4,5-\mathrm{P}_{3}$ représentent bien les récepteurs au moyen desquels
l'Ins $1,4,5-\mathrm{P}_{3}$ produit son effet lors d'une stimulation cellulaire, est de vérifier que l'Ins $1,4,5-\mathrm{P}_{3}$ est capable de provoquer la libération de $\mathrm{Ca}^{2+}$ dans les préparations microsomales où les sites de liaison sont observés. Dans la majorité des systèmes étudiés, l'Ins $1,4,5-\mathrm{P}_{3}$ stimule la libération $\mathrm{du}$ $\mathrm{Ca}^{2+}$, mais les concentrations nécessaires pour produire cet effet sont supérieures aux constantes d'affinité calculées dans les études de liaison (ce qui peut être expliqué, en partie, par la dégradation rapide de l'Ins 1, 4, 5$\mathrm{P}_{3}$ dans les conditions expérimentales utilisées pour mesurer la.libération de $\left.\mathrm{Ca}^{2+}\right)$. Il semble donc que le site de liaison pour l'Ins $1,4,5-\mathrm{P}_{3}$ (détecté dans les tissus périphériques) correspond à son récepteur physiologique. Afin d'appuyer cette interprétation, il a été démontré au niveau du cortex surrénalien que les activités de liaison de l'Ins 1,4,5- $\mathrm{P}_{3}$ et de libération de $\mathrm{Ca}^{2+}$ sont influencées de façon similaire par le dithiothréitol, le n-éthylmaléimide et le $\mathrm{pH}$; ce qui suggère qu'il s'agit bien de la même entité moléculaire [20].

\section{Localisation intracellulaire du site d'action de I'Ins 1,4,5-P $P_{3}$}

Jusqu'à maintenant, peu d'études ont tenté d'identifier l'organite intracellulaire possédant le récepteur de

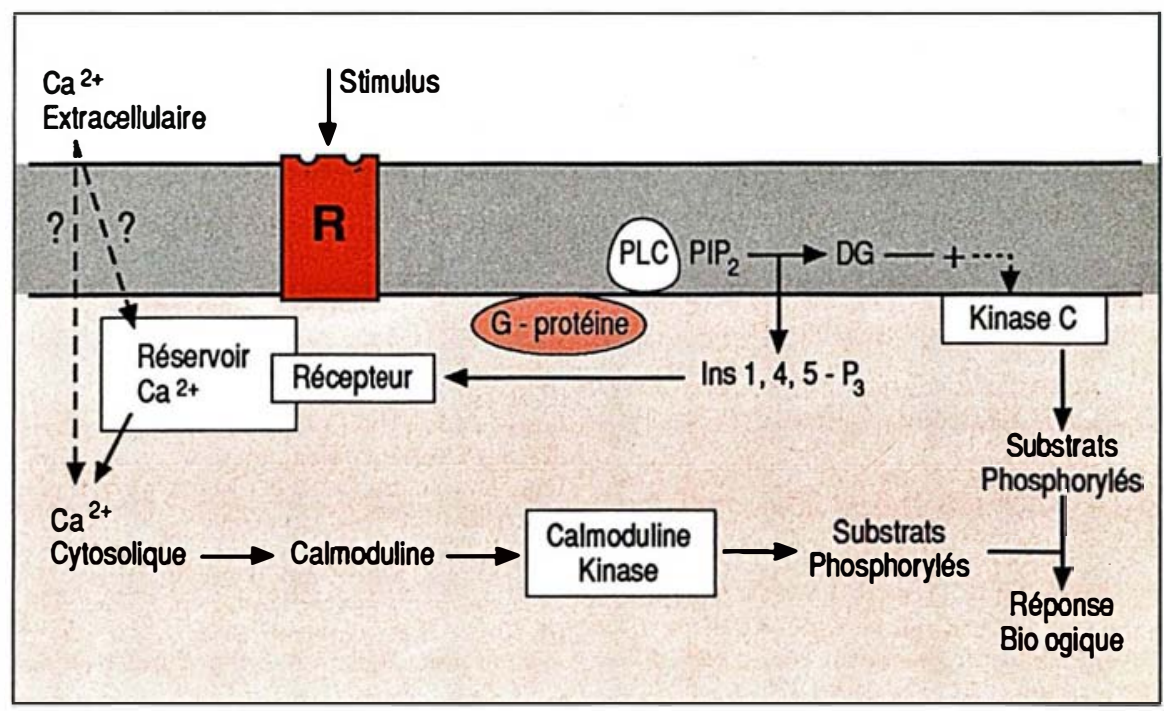

Figure 1. Mécanisme d'action des agents qui mobilisent le calcium. Les seconds messagers (Ins 1,4,5- $P_{3}$ et DG) produits par la phospholipase $C$ (PLC) activent (directement ou via des messagers intermédiaires) deux protéines kinases dont les actions combinées produisent l'effet biologique. 


\section{RÉFÉRENCES}

1. Berridge MJ. Inositol trisphosphate and diacylglycerol: two interacting second messengers. Ann Rev Biochem 1987 ; 56 : 159-93.

2. Williamson JR, Cooper RH, Joseph SK, Thomas AP. Inositol trisphosphate and diacylglycerol as intracellular second messengers in liver. Am J Physiol 1985 ; 248 : C203-16.

3. Abdel-Latif AA. Calcium-mobilizing receptors, polyphosphoinositides, and the generation of second messengers. Pharmacol Rev 1986 ; 38 : 227.72

4. Hokin LE, Hokin MR. Effects of acetylcholine on the turnover of phosphoryl units in individual phospholipids of pancreas slices and brain cortex slices. Biochem Biophys Acta 1955 ; 18 : 102-10.

5. Michell RH. Inositol phospholipids and cell surface receptor function. Biochem Biophys Acta $1975 ; 415$ : 81-147.

6. Nishizuka Y. The molecular heterogeneity of protein kinase $\mathrm{C}$ and its implications for cellular regulation. Nature 1988 ; 334 : 661-5.

7. Streb H, Irvine RF; Berridge MJ, Shulz I. Release of $\mathrm{Ca}^{2+}$ from a nonmitochondrial intracellular store in pancreatic acinar cells by inositol 1,4,5-trisphosphate. Nature 1983 ; $306: 67-9$.

8. Prentki $M$, Biden $T J$, Janjic $D$, Irvine RF, Berridge MJ, Wollheim CB. Rapid mobilization of $\mathrm{Ca}^{2+}$ from rat insulinoma microsomes by inositol 1,4,5-trisphosphate. Nature 1984 ; 309 : 562-4.

9. Gilman A. G-proteins and dual control of adenylate cyclase. Cell 1984 ; 36 : 577-9.

10. Nishizuka Y. The role of protein kinase $C$ in cell surface signal transduction and tumor promotion. Nature $1984 ; 308$ : 693-8.

11. Nishizuka Y. Studies and perspectives of protein kinase C. Science 1986 ; 233 : 305-12.

12. Carofoli E. Intracellular calcium homeostasis. Ann Rev Biochem 1987; 56: 395-433.

13. Baukal AJ, Guillemette G, Rubin R, Spat A, Catt KJ. Binding sites for inositoltrisphosphate in the bovine adrenal cortex. Biochem Biophys Res Commun 1985 ; 133 : 5328.

14. Spat A, Fabiato A, Rubin R. Binding of inositoltrisphosphate by a liver microsomal
15. Guillemette G, Balla T, Baukal A J, Catt KJ. Inositol 1,4,5-trisphosphate binds to a specific receptor and releases microsomal calcium in the anterior pituitary gland. Proc Natl Acad Sci USA 1987; 84 : 8195-9.

16. Spat A, Lukacs GL, Eberhardt I, Kie sel L, Runnebaum B. Binding of inositol phosphates and induction of $\mathrm{Ca}^{2+}$ release from pituitary microsomal fractions. Biochem J 1987 ; 244 : 493-6.

17. Spat A, Bradford P, McKinney JS, Rubin R, Putney JW. A saturable receptor for 32P-inositol-1,4,5-trisphosphae in hepatocytes and neutrophils. Nature 1986 ; 319: 514-6.

18. Vacca JP, De Solms SJ, Huff JR. Total synthesis of D- and L-myo-Inositol 1,4,5trisphosphate. J Am Chem Soc 1987; 109 : 3478-9.

19. Reese CB, Ward JG. Synthesis of D- myoInositol 1,4,5-trisphosphate. Tetrahedron Lett 1987 ; $28: 2309-12$

20. Guillemette G, Segui JA. Effects of $\mathrm{pH}$ reducing and alkylating reagents on the binding and $\mathrm{Ca}^{2+}$ rclcasc activities of inositol $\mathrm{l}$, 4,5 trisphosphate in the bovine adrenal cortex. Mol Endocrinol $1988 ; 2$ : 1249-55.

21. GuillemetteG, Balla T, Baukal A J Catt KJ. Characterization of inositol 1,4,5 trisphosphate receptors and calcium mobilization in a hepatic plasma membrane fraction. J Biol Chem 1988; 263 : 4541-8.

22. Henne V, Püper A, Soling HD. Inositol 1, 4, 5 trisphosphate and 5' - GTP induce calcium release from different intracellular pools. FEBS Lett 1987 ; 218 : 153-8.

23. Foskett JK, Gunter-Smith PJ, Melvin JE, Turner R J. Physiological localization of an agonist-sensitive pool of $\mathrm{Ca}^{2+}$ in parotid acinar cells. Proc Natl Acad Sci USA 1989; 86 : 167-71.

24. Dunlop ME, Larkins RG. GTP- and inositol 1, 4, 5-trisphosphate -induced release of ${ }^{45} \mathrm{Ca}^{2+}$ from a membrane store co-localized with pancreacti-islet-cell plasma membrane. Biochem J 1988 ; 253 : 67-72.

25. Volpe $\mathrm{P}$, Krause $\mathrm{KH}$, Hashimoto $\mathrm{S}$, et al. «Calciosome» a cytoplasmic organelle: The inositol 1,4,5-trisphosphate-sensitive $\mathrm{Ca}^{2+}$ store of nonmuscle cells? Proc Natl Acad Sci USA 1988 ; 85 : 1091-5.

26. Dawson AP. GTP enhances inositol tris phosphate-stimulated $\mathrm{Ca}^{2+}$ release from rat liver microsomes. FEBS Lett 1985 ; 185 : 147 150.
l'Ins 1,4,5-P $\mathrm{P}_{3}$. Dans le foie, les sites de liaison ont été retrouvés en majorité dans une fraction enrichie en membrane plasmique. La mobilisation de $\mathrm{Ca}^{2+}$ induite par l'Ins $1,4,5-\mathrm{P}_{3}$ est aussi plus importante dans cette fraction [21]. On en conclut que les réservoirs de $\mathrm{Ca}^{2+}$ sensibles à l'Ins 1,4,5$\mathbf{P}_{3}$ seraient une partie spécialisée du réticulum endoplasmique qui cosédimente ou est copurifiée avec la membrane plasmique lors du fractionnement cellulaire. On arriva à une conclusion semblable au moyen de préparations subcellulaires de glandes parotides dans lesquelles le réservoir de $\mathrm{Ca}^{2+}$ sensible à l'Ins 1,4,5- $\mathrm{P}_{3}$ était également copurifié avec la membrane plasmique [22, 23]. Une autre étude, utilisant des membranes plasmiques de cellules d'îlots pancréatiques a démontré que les réservoirs de $\mathrm{Ca}^{2+}$ sensibles à l'Ins 1,4,5- $\mathrm{P}_{3}$ étaient en effet associés à la membrane plasmique [24]. Enfin, une étude récente a mis en évidence la présence d'un nouvel organite dans le cytoplasme de cellules du foie et du pancréas [25]. Cet organite, nommé calciosome, peut accumuler de grandes quantités de $\mathrm{Ca}^{2+}$ grâce à son contenu riche en calséquestrine (une protéine qui contient environ 40 sites de liaisons de $\mathrm{Ca}^{2+}$ par mole). Etant donné que l'Ins $1,4,5-\mathrm{P}_{3}$ stimule la libération $\mathrm{du}$ $\mathrm{Ca}^{2+}$ à partir d'une préparation partiellement purifiée de ces organites, les auteurs suggèrent que les calciosomes représentent le site d'action de l'Ins $1,4,5-\mathrm{P}_{3}$ dans les tissus non musculaires. Bien que les conclusions de cette étude demandent à être confirmées, il est intéressant de noter que les tests immunohistochimiques ont localisé les calciosomes dans la région adjacente à la membrane plasmique.

\section{Mécanisme d'action de I'Ins 1,4,5- $P_{3}$}

Le récepteur de l'Ins 1,4,5- $\mathrm{P}_{3}$ contrôle-t-il l'activité d'un canal calcique ou est-il lui-même un canal calcique? Par analogie avec certains systèmes de transduction connus, on pourrait spéculer que le récepteur de l'Ins $1,4,5-\mathrm{P}_{3}$ est couplé à une protéine effectrice (le canal calcique) par l'intermédiaire d'une G-protéine. Ce modèle semblait tout à fait vraisem- 


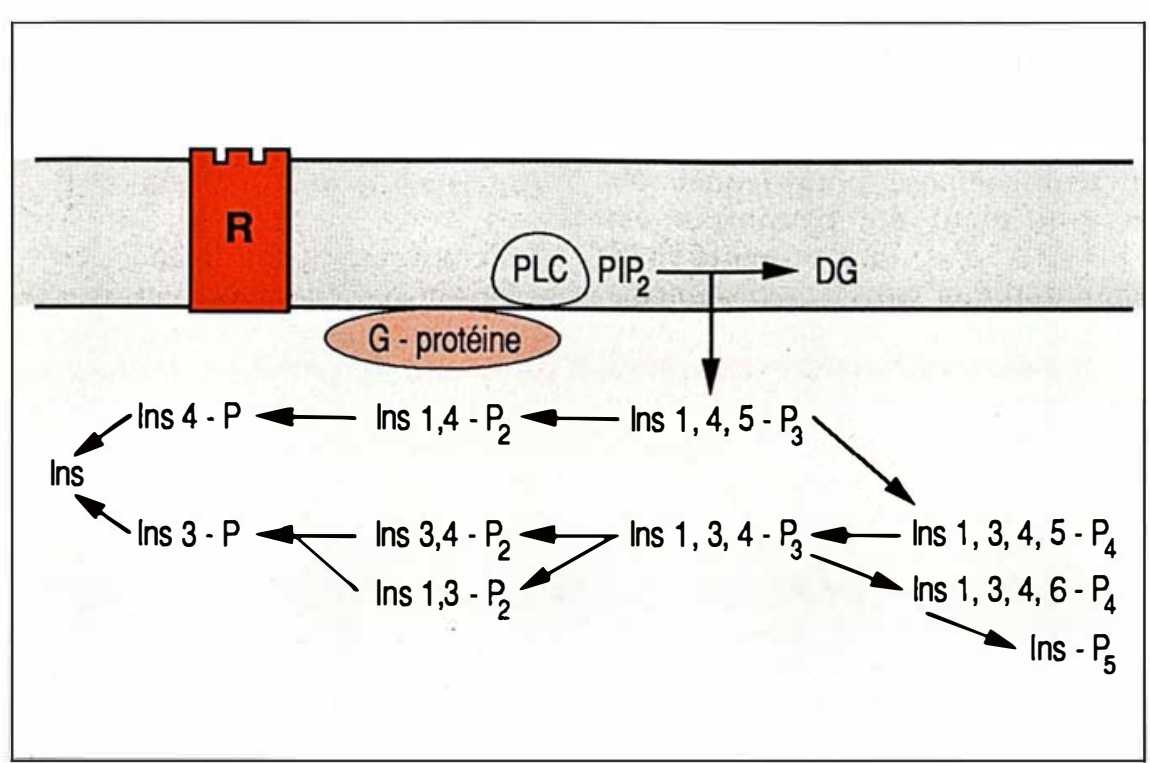

Figure 2. Production et métabolisme de l'Ins 1,4,5-P $P_{3}$ dans la cellule glomérulée du cortex surrénalien de bouf stimulée par l'angiotensine II. Des voies métaboliques similaires surviennent dans de nombreux types de cellules stimulées par des agents mobilisateurs du calcium.

blable à la suite des travaux originaux de Dawson qui avaient démontré que le GTP potentialise l'effet de l'Ins $1,4,5-\mathrm{P}_{3}$ sur la libération de $\mathrm{Ca}^{2+}[26]$. Mais après réévaluation de ce phénomène dans de nombreux laboratoires, il fut reconnu que les effets du GTP et de l'Ins 1,4,5- $\mathrm{P}_{3}$ étaient additifs et impliquaient des mécanismes distincts. A l'heure actuelle, les mécanismes moléculaires post-récepteur impliqués dans la libération du $\mathrm{Ca}^{2+}$ induite par l'Ins $1,4,5-\mathrm{P}_{3}$ sont encore mal définis. Les travaux de Smith et al. ont démontré que l'Ins $1,4,5-\mathrm{P}_{3}$ active un canal calcique, plutôt qu'un transporteur, par un mécanisme indépendant du métabolisme de l'ATP [27]. Ce canal calcique est distinct de la pompe calcique dépendante de l'ATP et permet l'efflux de $\mathrm{Ca}^{2+}$ dans le cytosol en échange d'un influx de $\mathrm{K}^{+}$. Le mécanisme le plus simple que l'on puisse suggérer serait que l'Ins $1,4,5-\mathrm{P}_{3}$, en se liant à son récepteur spécifique, ouvre un canal ionique à la manière du récepteur cholinergique nicotinique.

Des études électrophysiologiques ont confirmé que l'Ins 1,4,5- $\mathrm{P}_{3}$ ouvre un canal calcique. Ehrlich et Watras ont observé cet effet sur des vésicules de réticulum sarcoplasmique de muscle $\mathrm{m} / \mathrm{s} \mathrm{n}^{\circ} 8$ vol. 5 , octobre 89
l'Ins 1,4,5- $\mathrm{P}_{3}$ contrôlerait à la fois la mobilisation intracellulaire de $\mathrm{Ca}^{2+}$ et l'influx du $\mathrm{Ca}^{2+}$ extracellulaire. Ce modèle est d'autant plus attrayant qu'il suggère une façon simple de manipuler le gradient fantastique de $\mathrm{Ca}^{2+}$ qui existe entre le cytosol $(0,1 \mu \mathrm{M})$ et l'extérieur de la cellule $(1,0 \mathrm{mM})$; le réservoir intracellulaire servant d'antichambre ou de zonetampon entre les deux milieux.

\section{Rôle des autres inositolphosphates}

Lors de la stimulation cellulaire, la phospholipase $\mathrm{C}$ hydrolyse sélectivement le $\mathrm{PIP}_{2}$ (au moins pendant les premières minutes). Les produits dérivant de ce substrat sont donc le DG et l'Ins 1,4,5- $\mathrm{P}_{9}$. L'analyse des contenus intracellulaires, suite à de courtes périodes de stimulation, démontre cependant la formation de nombreux autres inositolphosphates. L'Ins $1,4,5-\mathrm{P}_{3}$ est, en fait, très rapidement métabolisé par des enzymes qui lui enlèvent ou lui ajoutent des groupements phosphates. La figure 2 montre la cascade d'événements survenant lors de la stimulation de cellules glomérulées du cortex surrénalien de bouf par l'angiotensine II. En bref, l'Ins 1,4,5$\mathrm{P}_{3}$ peut être déphosphorylé par une phosphatase spécifique agissant en position 5 pour produire l'Ins 1,4,5$\mathrm{P}_{2}$. Ce dernier produit sera alors déphosphorylé davantage en Ins 4-P et enfin en inositol libre. L'Ins 1,4,5$\mathrm{P}_{3}$ peut aussi être phosphorylé en position 3 par une kinase spécifique qui produit l'Ins 1,4,5- $\mathrm{P}_{4}$ qui, lui, est déphosphorylé en Ins $1,3,4-P_{3}$. Tout récemment, on a découvert une nouvelle kinase qui transforme l'Ins $1,3,4-\mathrm{P}_{3}$ en Ins $1,3,4,6-\mathrm{P}_{4}$ [32] et, dans la même série d'études, il a été démontré que l'Ins 1,3,4,6- $\mathrm{P}_{4}$ est le précurseur d'un produit plus polaire (Ins- $P_{5}$ ) de structure non encore identifiée. Aucun de ces composés hautement phosphorylés ne s'accumule à l'intérieur de la cellule et il faut donc présumer qu'ils sont les cibles de phosphatases qui les hydrolysent séquentiellement.

Si l'origine de tous ces inositolphosphates est assez bien définie, leur rôle, pour la plupart, demeure inconnu. A part l'Ins $1,4,5-\mathrm{P}_{3}$ (dont le rôle a été décrit précédemment) et 


\section{RÉFÉRENCES}

27. Smith JB, Smith L, Higgins BL. Temperature and nucleotide dependence of calcium release by myo-inositol 1,4,5-trisphosphate in cultured vascular smooth muscle cells. J Biol Chem 1985 ; 260 : 14413-6.

28. Ehrlich BE, Watras J. Inositol 1,4,5-trisphosphate activates a channel from smooth muscle sarcoplasmic reticulum. Nature 1988 ; 336 : 583-6.

29. Kuno M, Gardner P. Ion channels activated by inositol 1,4,5-trisphosphate in plasma membrane of human T-lymphocytes. Nature $1987 ; 326: 301-4$

30. Vilven J, Coronado R. Openning of dihydropyridine calcium channels in skeletal muscle membranes by inositol trisphosphate. Nature 1988 ; 336 : 587-9.

31. Putney JW. A model for receptor regulated calcium entry. Cell Calcium 1986; $7: 1$ 12.

32. Balla T, Baukal A J, Guillemette G, Catt KJ. Multiple pathways of inositol polyphosphate metabolism in angiotensin-stimulated adrenal glomerulosa cells. J Biol Chem 1988 ; 263 : 4083-91.

33. Irvine R F, Moore RM. Micro-injection of inositol 1,3,4,5-tetrakisphosphate activates sea urchin eggs by a mechanism dependent on external $\mathrm{Ca}^{2+}$. Biochem J 1986; $240: 917$. 20.

34. Crossley I, Swann K, Chambers E, Whitaker M. Activation of sea urchin eggs by inositol phosphates is independent of external calcium. Biochem J 1988; 252 : 257-62.

35. Hill TD, Dean NM, Boynton AL. Inositol 1,3,4,5-tetrakisphosphate induced $\mathrm{Ca}^{2+}$ sequestration in rat liver cells. Science 1988 ;
l'Ins 1,4- $\mathrm{P}_{2}$, qui serait un métabolite inactif (donc l'hydrolyse du phosphate en position 5 serait un moyen pour la cellule de terminer l'action), le seul composé pour lequel des actions aient été proposées est l'Ins 1,3,4,5-P $\mathrm{P}_{4}$. On a rapporté qu'il serait impliqué dans les mouvements transmembranaires de $\mathrm{Ca}^{2+}$ chez l'œuf d'oursin [33], mais ces résultats ont été contestés par une étude récente [34]. Il a aussi été rapporté que l'Ins 1,3,4,5- $\mathrm{P}_{4}$ stimule en synergie avec l'Ins $1,4,5-\mathrm{P}_{3}$ là relâche du $\mathrm{Ca}^{2+}$ d'une préparation microsomale de cellules hypophysaires [16]. En revanche, avec une préparation microsomale de foie de rat, Hill et al. ont obtenu des résultats suggérant un rôle tout à fait opposé pour l'Ins $1,3,4,5-\mathrm{P}_{4}$, qui diminuerait la concentration cytosolique de $\mathrm{Ca}^{2+}$ en stimulant sa recapture dans les réservoirs intracellulaires [35]. Il semble donc que ce composé soit impliqué dans l'homéostasie calcique, mais il est clair que de nouvelles études sont nécessaires avant d'aboutir à un consensus sur son rôle et son mode d'action.

Quant aux autres inositol phosphates (dont quelques-uns sont de découverte très récente), aucune fonction ne leur a encore été attribuée. La synthèse de ces composés requiert la présence d'enzymes spécifiques et utilise des voies métaboliques dont certaines étapes de phosphorylation dépendantes de l'ATP consomment de l'énergie cellulaire. A moins que cette dépense d'énergie ne soit futile, il faut supposer que certains de ces composés ont un rôle à jouer à l'intérieur de la cellule.

Ainsi, dans ce mécanisme d'action où la phospholipase $\mathrm{C}$ est l'enzyme clé, alors que les effets de l'Ins 1,4,5$\mathrm{P}_{3}$ et du DG sont bien reconnus, les aspirants au titre de messager intracellulaire sont nombreux

Remerciements

G. Guillemette est un chercheur-boursie du Fonds de la recherche en santé du Québec. Les travaux de laboratoire sont financés par le Conseil de recherches médicales du Canada.

\section{Summary}

Intracellular messengers generated by phospholipase $\mathbf{C}$

Calcium mobilizing ligands activate phospholipase $C$, thus generating inositol 1,4,5-trisphosphate and diacylglycerol, two second messengers whose role and mechanism of action are well characterized. Inositol 1,4,5-trisphosphate binds to specific intracellular receptors which control the activity of a calcium channel to provoque calcium release from a cell-associated, non-mitochondrial pool. Diacylglycerol activates protein kinase C. Once generated during cell stimulation, inositol 1,4,5-trisphosphate undergoes a series of phosphorylation and dephosphorylation steps, thus generating numbers of different inositol phosphates whose physiological relevances are still not well known.

PRIX NOBEL DE MÉDECINE 1989 POUR LA DÉCOUVERTE DES ONCOGÉNES CELLULAIRES A MICHAEL BISHOP ET HAROLD VARMUS. La première référence du premier éditorial de $\mathrm{m} / \mathrm{s}$ (no 1, vol. 1, p. 10) était : Stehelin D., Varmus HE, Bishop JM, Vogt PK. Nature 1976; 260 : 170-3... les auteurs qui ont découvert que, aux oncogènes viraux correspondaient des oncogènes cellulaires. Varmus et Bishop viennent de se voir décerner le prix Nobel... et pas le premier auteur, Dominique Stehelin, qui effectuait alors dans leur laboratoire un stage postdoctoral et qui a joué un rôle essentiel dans cette découverte !

TIRÉS A PART

G. Guillemette 